\title{
Trends in demographics and outcome of patients presenting with traumatic brain injury
}

\author{
Rachel Kadar' ${ }^{1}$, Daniel Rochford', Ellen Omi ${ }^{1}$, Yalaunda Thomas ${ }^{1}$, \\ Kunal Patel ${ }^{2}$, Erik Kulstad ${ }^{3}$ \\ ${ }^{1}$ Department of Emergency Medicine, Advocate Christ Medical Center, Oak Lawn, IL, USA \\ ${ }^{2}$ MetroHealth Medical Center, Cleveland, OH, USA \\ ${ }^{3}$ Department of Emergency Medicine, UT Southwestern Medical Center, Dallas, TX, USA
}

Objective To analyze the trends in demographics and outcomes of patients presenting with traumatic brain injury by performing a retrospective database review of the Illinois Department of Public Health (IDPH) Trauma Registry.

Methods We utilized the IDPH Trauma Registry to retrieve data on patients treated for traumatic brain injuries at our large, tertiary care hospital from 2004 to 2012, inclusive. From this data, logistic regression models were used to analyze and compare basic demographics such as age, sex, and clinical outcome.

Results Three thousand and thirty-nine patients were analyzed with a mean age of 43 (standard deviation, 24) and a median age of 41 (interquartile range, 23 to 60). Over the study period, patients' age increased steadily from 32 to 49 years. The percentage of female patients increased, from $16.4 \%$ to $27.5 \%$ over the last 4 years. Overall mortality was greater for males than females (22.1\% vs. $17.3 \%$; odds ratio [OR], 1.36; 95\% confidence interval [CI], 1.10 to 1.68 ). Mortality decreased over the period $(\mathrm{OR}, 0.88 ; 95 \% \mathrm{Cl}, 0.85$ to 0.91$)$, with a greater decrease in females ( $\mathrm{OR}, 0.84 ; 95 \% \mathrm{Cl}, 0.78$ to 0.90$)$ than in males $(\mathrm{OR}, 0.90 ; 95 \% \mathrm{Cl}, 0.86$ to 0.94$)$.

Conclusion Although the age of patients presenting with traumatic brain injury is increasing substantially, the data suggests that overall mortality appears to be decreasing, and this decrease appears to be greater in females than in males. These changes in trends found in the IDPH Trauma Registry supports the importance for further analysis of other reliable public datasets to identify areas of future study.

Keywords Brain injuries, traumatic; Patient outcome assessment; Demography; Emergency service, hospital
eISSN: 2383-4625

Received: 10 February 2018

Revised: 17 May 2018

Accepted: 24 May 2018

Correspondence to: Daniel Rochford Department of Emergency Medicine, Advocate Christ Medical Center, 4440 West 95th Street, Oak Lawn, IL 60453, USA

E-mail: Daniel.Rochford@ advocatehealth.com

ORCID

http://orcid.org/0000-0002-0122-0924

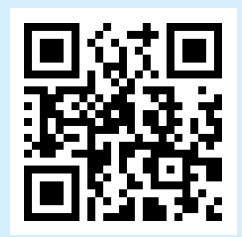

How to cite this article:

Kadar R, Rochford D, Omi E, Thomas Y, Patel $\mathrm{K}$, Kulstad E. Trends in demographics and outcome of patients presenting with traumatic brain injury. Clin Exp Emerg Med 2019;6(2):113-118.

This is an Open Access article distributed under the terms of the Creative Commons Attribution Non-Commercial License (http:// creativecommons.org/licenses/by-nc/4.0/). 


Capsule
What is already known
Traumatic brain injury (TBI) is a major cause of death and disability, with over 1.1 million emergency department visits,
235,000 hospital admissions, and 50,000 deaths annually in the US. Prior studies on TBI rely on large public datasets
that have recently shown limitations in data reliability. One recent study has shown that the Illinois Department of
Public Health (IDPH) Trauma Registry has infrequent incongruent dispositions, which suggests high reliability. It is for
this reason that the IDPH Trauma Registry was utilized in this study to further explore the temporal trends in TBI.
What is new in the current study
Although the age of patients presenting with TBI is increasing substantially, the data suggests that overall mortality
appears to be decreasing, and this decrease appears greater in females than in males. These changes in trends found in
the IDPH Trauma Registry support the importance of further analysis of other reliable public datasets to identify areas
of future study.

\section{INTRODUCTION}

Traumatic brain injury (TBI) is a major cause of death and disability, with over 1.1 million emergency department visits, 235,000 hospital admissions, and 50,000 deaths occurring annually in the United States alone. In addition, 30.5\% of all injury deaths include a diagnosis of TBI. ${ }^{1,2}$ The prevalence of long-term disability due to TBI in the United States is between 3.2 to 5.3 million, with lifetime medical costs in 2010 estimated at $\$ 11.5$ billion. ${ }^{1,3}$ In addition to functional and cognitive impairment, disabilities extend to include psychological stressors, such as increased irritability, anxiety, depression, and suicidal ideation. ${ }^{4,5}$

Traditionally, rates of TBI have been thought to be highest in adolescents and young adults, although an increase in the elderly (age $>65$ ) has been observed. Among adults aged 65 and older, there was an increase in fall-related TBls from 2002 to 2006, with a $46 \%$ increase in emergency department visits, a $34 \%$ increase in hospitalizations, and a $27 \%$ increase in death. ${ }^{6}$ Therefore, with the increase in the average age in the US, the demographics of TBI may be evolving, which will impact outcome and management strategies.

Previous studies have shown large public datasets, such as the NHAMCS (National Hospital Ambulatory Medical Care Survey), have disposition discrepancies, which reflects a measure of their internal validity. As a result, these datasets, which are widely used for research, may have significant limitations in data reliability. ${ }^{7,8}$ Since many prior studies on TBI rely on these datasets, analysis of trends and outcomes in other databases would be helpful to better understand areas in need of further study.

One recent study showed that the Illinois Department of Public Health (IDPH) Trauma Registry has infrequent incongruent dispositions, a finding suggesting high reliability. ${ }^{9}$ Studies suggest that unreliable datasets may be due to insufficient abstractor training, and the IDPH Trauma Registry abstractors are required to have both medical and abstractor training, which may contribute to the quality of this localized data. ${ }^{9}$ To further explore temporal trends in TBI using a high-reliability dataset, we sought to examine the IDPH Trauma Registry to determine changes in demographics and outcome of patients, in Illinois, presenting with TBI over a 9-year timeframe.

\section{METHODS}

\section{Study design and setting}

This is a retrospective database review, analyzing data from the IDPH Trauma Registry. Data included in this study captures patients treated for TBI at a Level 1 Trauma Center and large tertiary care hospital by the trauma service during the years 2004 to 2012, inclusive. The dataset includes all patients with traumatic injuries that were seen and admitted to the trauma service. Specifically, this includes patients that were transferred to the trauma center and subsequently admitted, including patients that were dead on arrival or died in the emergency department. Patients that died on the scene of the traumatic injury but were not transported to a trauma center, and patients who were treated in the emergency department and discharged in less than 12 hours were not included in the database. Criteria to be seen by the trauma service on arrival in the emergency department were initiated in the field and includes: patients with a head injury with persistent unconsciousness or focal signs such as seizures; posturing or the inability to respond to simple commands; transmediastinal gunshot wounds; spinal cord injury with paralysis; maternal trauma with significant mechanism and/or obvious trauma at 20 to 32 weeks gestation; pediatric trauma including blunt or penetrating head, chest, or abdominal trauma. For blunt or penetrating trauma, patients with unstable vital signs (systolic blood 
pressure $<90 \mathrm{mmHg}$ ), respiratory compromise (respiratory rate $<10$ or $>29$ breaths/min), or head injury with Glasgow Coma Score $\leq 10$ were included. The Glasgow Coma Scale is used to categorize traumatic head injuries based on severity. Total scores of 13 to 15 is defined as mild, 9 to 12 as moderate, 3 to 8 as severe. ${ }^{10}$ For anatomical injury, penetrating injury of the head, neck, chest or abdomen, two or more body regions with potential life or limb threat, amputation above the wrist or ankle, limb paralysis above the wrist or ankle, flail chest, or two or more proximal long bone fractures were included. For mechanism of injury, ejection from a motor vehicle, death in the same passenger compartment, falls $>20$ feet, falls $>3$ times the body length (for children), and maternal trauma $>24$ weeks were included. For trauma transfers, patients with a head injury as seen on computed tomography imaging (subdural hematoma, subarachnoid hemorrhage, intraparenchymal hemorrhage) with a Glasgow Coma Scale $<9$, or if the transferring physician felt the patient would require intensive care unit level of care were included.

This study was institutional review board approved and granted a waiver of the consent process since the data was publicly available and de-identified.

\section{Methods of measurement}

Basic demographics, such as age and sex, and clinical outcomes from over 3,000 patients were exported from the database into Excel. Outcomes, such as ventilator days, hospital admission days, intensive care unit days, Glasgow Coma Scale and mortality were compared across time and age. Data points were grouped by quarter to provide a reasonable sample size per time period. This data was analyzed and compared over the years with logistic regression models which used age, sex, and clinical outcomes as variables.

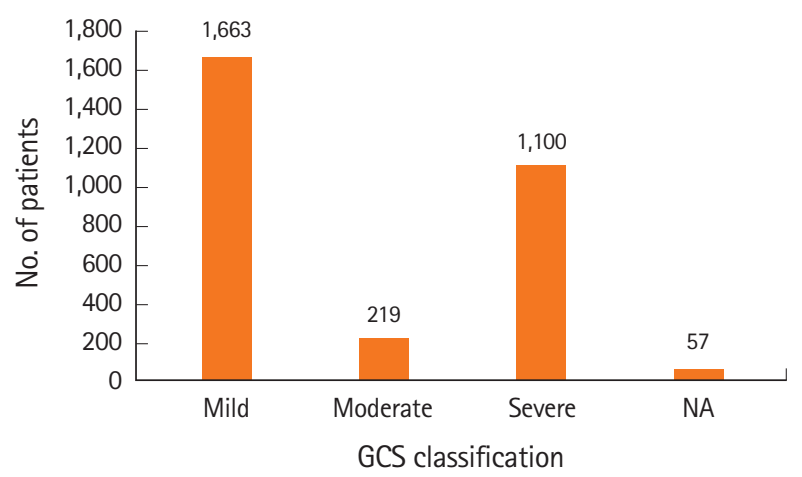

Fig. 1. Classification of traumatic brain injury severity based on the Glasgow Coma Scale (GCS) score from 3,039 patients spanning 2004 to 2012. Of the 3,039 patients, 1,663 (55\%) were classified as sustaining mild traumatic brain injury, 219 (7\%) were moderate, 1,100 (36\%) were severe, and 57 (2\%) of the patients did not receive scores. NA, not available.

\section{Outcome measures}

Clinical outcomes were recorded as one of three categories: discharged, morgue, or other. An outcome of 'discharged' consisted of several dispositions including: transferred to another hospital or subacute hospital, discharged home or self-care, discharged to child protection agency, discharged to nursing home/skilled nursing facility/intermediate care facility/residential, discharged to jail/prison, discharged to a psychiatric hospital, discharged to a rehabilitation center, and left against medical advice. An outcome of 'morgue' was any patient that did not survive to discharge. This includes patients that were sent to the morgue, funeral home, medical examiner or coroner. An outcome of 'other' was given to those whose discharges were unknown. This category only accounted for $0.07 \%$ of the patients that were analyzed, and therefore was not included on figures.

\section{RESULTS}

A total of 3,039 patients with TBI were treated over the study period. TBI was defined as having an International Classification of Diseases 9th revision diagnosis code under the category of intracranial injury and/or skull fracture. Based on the Glasgow Coma Scale, 1,663 (55\%) of the patients with TBI were classified as mild, $219(7 \%)$ were moderate, 1,100 (36\%) were severe, and $57(2 \%)$ of the patients did not receive scores (Fig. 1). The patients' ages ranged from less than 1 month to 109 years of age. The mean age of the study sample was 43 years (standard deviation, 24) and the median age was 41 (interquartile range, 23 to 60) (Fig. 2). Three peaks in age can be visualized in this histogram (Fig. 2), including young adult, middle aged, and elderly. Age increased steadily throughout the study period, with a mean age of

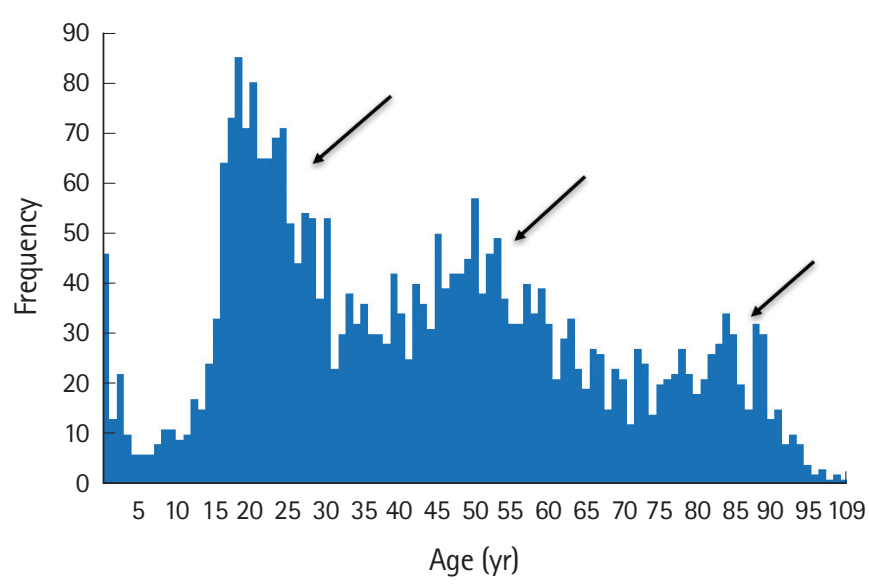

Fig. 2. Sample size and distribution by age as recorded in the Illinois Department of Public Health Trauma registry. Arrows were used to highlight three peaks in age: young adult, middle aged, and elderly. 


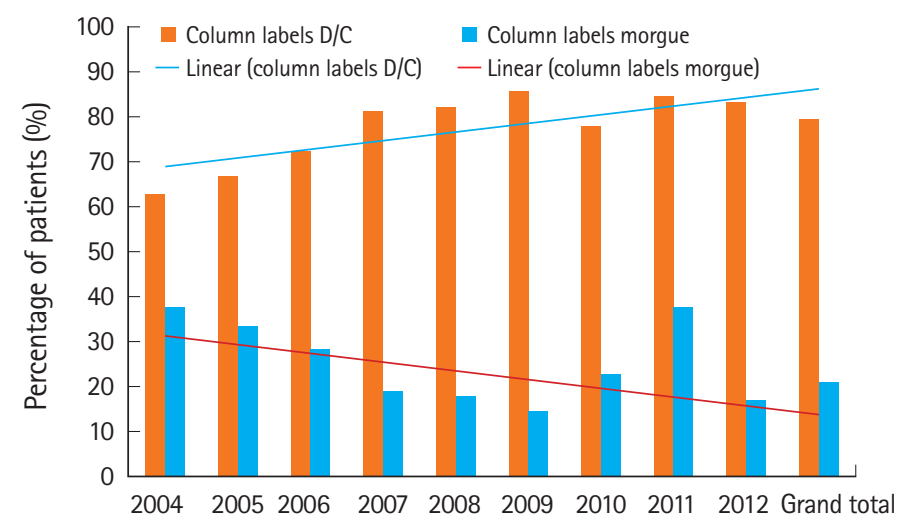

Fig. 3. Percentage of patients by outcome as seen by mortality and discharge rates $(\mathrm{D} / \mathrm{C})$ trended over time. Mortality decreased over the course of the study while $\mathrm{D} / \mathrm{C}$ increased. Overall mortality was $21 \%$ while the overall discharge rate was $79 \%$.

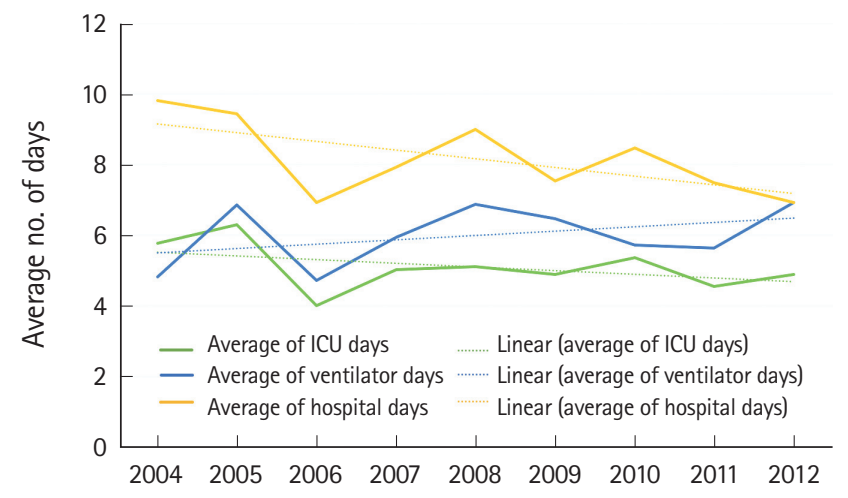

Fig. 4. Average number of ventilator days, intensive care unit (ICU) days, and hospital days trended over time. These trends did not show a significant change over the study period.

32 years in 2004 and a mean of 49 years in 2012. Overall mortality was $21 \%$. Patient mortality decreased over the entire period (odds ratio [OR], 0.88; 95\% confidence interval [Cl], 0.85 to 0.91) (Fig. 3). Ventilator days, intensive care unit days, and hospital admission days did not show a significant change over time (Fig. 4). Glasgow Coma Scale scores, after the first 2 years, remained stable over time (Fig. 5).

Twenty-five percent of the patients in the study period were female. The percentage of female TBI patients increased throughout the study period, from $16.4 \%$ initially, to $27.5 \%$ over the last 4 years (Fig. 6). In addition, a greater decrease in mortality was seen in females $(\mathrm{OR}, 0.84 ; 95 \% \mathrm{Cl}, 0.78$ to 0.90$)$ than in males (OR, 0.90; 95\% Cl, 0.86 to 0.94) (Fig. 7).

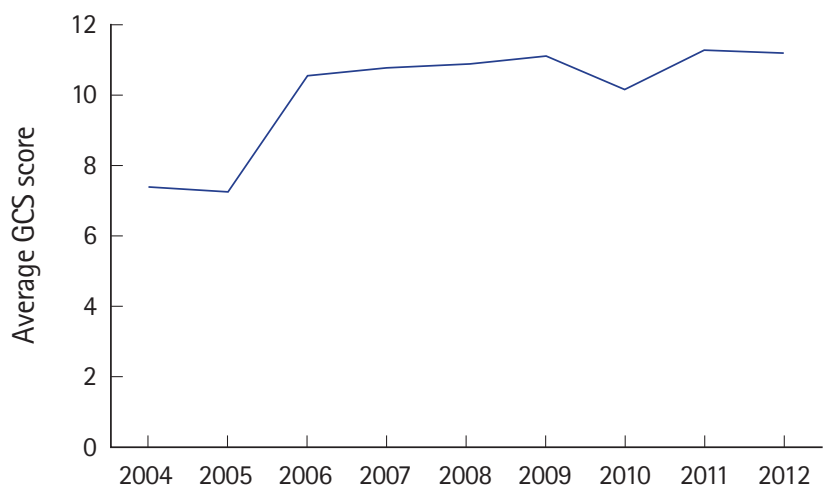

Fig. 5. Average Glasgow Coma Scale (GCS) score across time. Scores remained stable after the first 2 years.

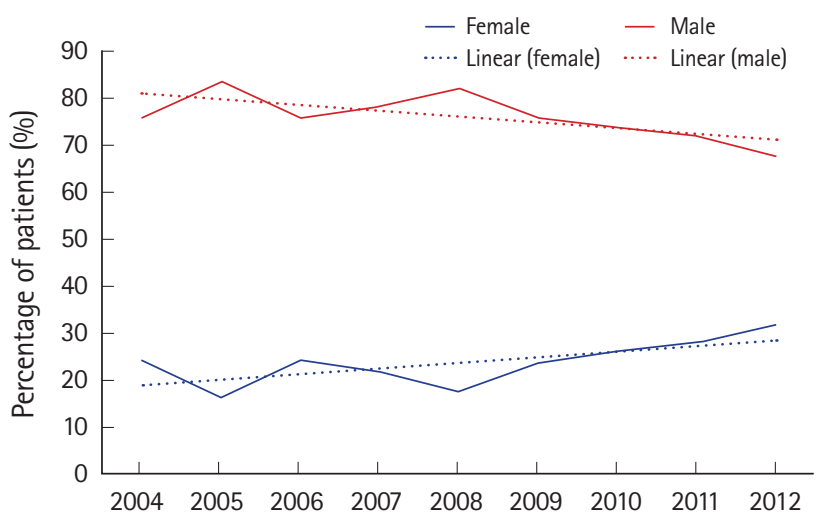

Fig. 6. Sex distribution of traumatic brain injuries over time. From 2009 to 2012 , the percentage of females increased from $16.4 \%$ to $27.5 \%$.

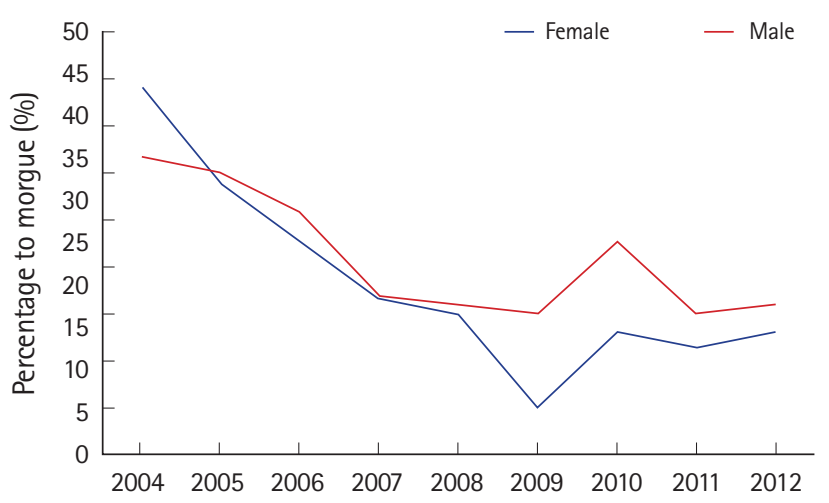

Fig. 7. Percent mortality by sex over time. Females showed a greater decrease in percent mortality than males over the study period.

\section{DISCUSSION}

From 2004 to 2012, the average age of patients treated for TBI at our Level 1 Trauma Center increased over time, which was accompanied by an increased percentage of female sex. Despite these temporal trends, mortality improved for both sexes, with a 
greater improvement for females. These changes in trends and outcomes of TBI in the IDPH Trauma Registry support the importance of analyzing other databases to identify areas for further study.

Given the aging patient population and likelihood of comorbidities, one may expect that an increase in mortality would be concurrently seen. However, unexpectedly, the overall percent mortality decreased over time. This could be due to many factors, including a decrease in severity of injury presentation or an improvement of patient care; however, based on Glasgow Coma Scale score, our population injury severity appeared to have remained relatively stable over the majority of our period of analysis. A possible unmeasured decrease in injury severity at presentation, due to factors such as improved pre-hospital care or change in safety rules/public awareness, may account for these findings. Emergency medical services and pre-hospital treatment, as well as in-hospital treatment and rehabilitation, continues to improve, which provides better outcomes for TBI. ${ }^{11,12}$

Illinois does not currently implement a helmet law for motorcyclists and there were no changes in helmet laws during the study period. However, some changes to legislature regarding seatbelt laws in Illinois were implemented. In 2003, Illinois implemented a primary seatbelt law, allowing police to pull over motorists to ticket them for not wearing a seatbelt. On January 1 , 2012, all backseat passengers in Illinois were required to wear a seatbelt, not just those under 19 years of age as was previously mandated. A decrease in mortality may also be due to improved in-hospital care. However, the lack of a substantial decrease in intensive care unit, hospital, and ventilator days with the change in mortality may suggest that there is no significant change in severity of injury or hospital management of these patients.

Regarding sex differences in mortality, there has been some speculation that progesterone may be protective in acute brain injury. A recent study involving mice found that females treated with sex steroids (progesterone and estrogen) showed a decreased sensitivity to TBI compared to male and female mice that lack the sex steroids. ${ }^{13}$ However, recent clinical investigations have concluded that the administration of progesterone did not decrease mortality in patients presenting with $\mathrm{TB} .{ }^{14}$ Other investigators have proposed multiple factors that may contribute to the negative results in clinical trials such as the overall complexity in the pathophysiology of TBI, pre-existing conditions, and the difficulty in controlling for other confounding variables compared to animal models. ${ }^{15,16} \mathrm{~A}$ third factor proposed is suboptimal dosing parameters from pre-clinical trials which may have led to too high a drug dose over too short of an administration period during the clinical investigation. ${ }^{17}$ Due to these factors, further re- search is required to understand the potential role progesterone has on both the outcome and treatment of TBI.

The overall quality of research on traumatic brain injuries is dictated by the reliability of the datasets that the researchers choose to use. Prior studies on TBI rely on large public datasets that have recently shown limitations in data reliability. Without reliable data, studies on the topic of TBI runs an increased risk of poorly representing the broader scope of the injury as well as the individuals affected by it. When this occurs, the progress in identifying and treating traumatic brain injuries is slowed. Analyzing databases such as the IDPH Trauma Registry has shown to be beneficial in understanding the trends and demographics of TBI. It is for this reason that researchers should place emphasis on datasets that incorporate rigorous training methods to minimize the amount of discrepancies and produce the most accurate data possible. Applying the use of these datasets can be beneficial in determining whether the changes in epidemiology of TBI found in this study are congruent with rates at the state and national level.

This study has the inherent limitations associated with retrospective database review. In addition, this analysis, as is common with many analyses of $\mathrm{TBI}$, does not sub-categorize type of TBI. International Classification of Diseases 9th revision codes are non-specific, which includes a variety of codes such as 'cortex contusion' to 'subdural hemorrhage' to 'skull fracture.' It also does not stratify by cause of death, nor does it distinguish among various levels of neurological outcomes.

Although the age of patients presenting with TBI is increasing substantially, the data suggests that overall percent mortality appears to be decreasing, and this decrease appears greater in females than in males. These trends in demographics and outcomes of TBI found in the IDPH Trauma Registry supports the importance for further analysis of other reliable public datasets to identify areas of future study.

\section{CONFLICT OF INTEREST}

No potential conflict of interest relevant to this article was reported.

\section{REFERENCES}

1. Centers for Disease Control and Prevention (CDC). CDC grand rounds: reducing severe traumatic brain injury in the United States. MMWR Morb Mortal Wkly Rep 2013;62:549-52.

2. Langlois JA, Rutland-Brown W, Wald MM. The epidemiology and impact of traumatic brain injury: a brief overview. J Head Trauma Rehabil 2006;21:375-8. 
3. Zaloshnja E, Miller T, Langlois JA, Selassie AW. Prevalence of long-term disability from traumatic brain injury in the civilian population of the United States, 2005. J Head Trauma Rehabil 2008;23:394-400.

4. Reeves RR, Laizer JT. Traumatic brain injury and suicide. J Psychosoc Nurs Ment Health Serv 2012;50:32-8.

5. Yang CC, Huang SJ, Lin WC, Tsai YH, Hua MS. Divergent manifestations of irritability in patients with mild and moderateto-severe traumatic brain injury: perspectives of awareness and neurocognitive correlates. Brain Inj 2013;27:1008-15.

6. Faul $M, X u L$, Wald $M$, Coronado V. Traumatic brain injury in the United States: emergency department visits, hospitalizations and deaths 2002-2006 [Internet]. Atlanta, GA: Centers for Disease Control and Prevention; 2010 [cited 2018 May 19]. Available from: http://www.cdc.gov/traumaticbraininjury/pdf/ blue_book.pdf.

7. Green SM. Congruence of disposition after emergency department intubation in the National Hospital Ambulatory Medical Care Survey. Ann Emerg Med 2013;61:423-6.

8. McCaig LF, Burt CW, Schappert SM, et al. NHAMCS: does it hold up to scrutiny? Ann Emerg Med 2013;62:549-51.

9. Kerem Y, Omi EC, Kulstad CE, Kulstad EB. Congruency of disposition after emergency department intubation in a regional database. Ann Emerg Med 2015;65:232-3.

10. Teasdale G, Jennett B. Assessment of coma and impaired con- sciousness: a practical scale. Lancet 1974;2:81-4.

11. Gerber LM, Chiu YL, Carney N, Hartl R, Ghajar J. Marked reduction in mortality in patients with severe traumatic brain injury. J Neurosurg 2013;119:1583-90.

12. Rosenfeld JV, Maas Al, Bragge $P$, Morganti-Kossmann MC, Manley GT, Gruen RL. Early management of severe traumatic brain injury. Lancet 2012;380:1088-98.

13. Clevenger AC, Kim H, Salcedo $E$, et al. Endogenous sex steroids dampen neuroinflammation and improve outcome of traumatic brain injury in mice. J Mol Neurosci 2018;64:410-20.

14. Goldstein FC, Caveney AF, Hertzberg VS, et al. Very early administration of progesterone does not improve neuropsychological outcomes in subjects with moderate to severe traumatic brain injury. J Neurotrauma 2017;34:115-20.

15 . Wright DW, Yeatts SD, Silbergleit $R$, et al. Very early administration of progesterone for acute traumatic brain injury. $\mathrm{N}$ Engl J Med 2014;371:2457-66.

16. Skolnick BE, Maas AI, Narayan RK, et al. A clinical trial of progesterone for severe traumatic brain injury. N Engl J Med 2014; 371:2467-76.

17. Howard RB, Sayeed I, Stein DG. Suboptimal dosing parameters as possible factors in the negative phase III clinical trials of progesterone for traumatic brain injury. J Neurotrauma 2017; 34:1915-8. 